\title{
Avaliaçáo do Conhecimento dos Fisioterapeutas Neurofuncionais acerca da Síndrome de Pusher
}

\author{
Evaluation of the Knowledge about Pusher Syndrome in Neurofunctional Physiotherapists
}

\section{Caroline Micaela Santos Góis ${ }^{1}$, Marta Caroline Nascimento Vilar de Araújo ${ }^{1}$, Karina de Carvalho da Silva ${ }^{2}$, Alessandra Teixeira da Câmara Araújo 3}

\begin{abstract}
RESUMO
Objetivo. Analisar o nível de conhecimento dos fisioterapeutas neurofuncionais acerca da Síndrome de Pusher (SP). Método. Aplicou-se um questionário com os fisioterapeutas que realizam atendimentos neurofuncionais em clínicas, hospitais e serviços de home care em Recife-PE. Resultados. Dos 60 entrevistados, somente 37 conheciam a SP. Deste universo de 37, 40.5\% já realizaram alguma pesquisa sobre a SP. Todos os fisioterapeutas apontaram o AVC como a principal causa; porém, menos de 35\% indicaram tumores cerebrais e traumatismo cranioencefálico. O tálamo póstero-lateral foi indicado por $35.1 \%$ deles como a possível estrutura lesada. As posturas ortostática (62.2\%) e sentada (21.6\%) foram informadas como a que mais se observa o comportamento peculiar da SP. $62.2 \%$ relataram que o paciente resiste à correção da sua postura por insegurança. As principais características clínicas relatadas foram o deslocamento do centro de gravidade para o lado plégico $(59.5 \%)$ e a utilização do hemicorpo sadio para se empurrar (51.4\%). Por fim, os recursos terapêuticos mais utilizados foram: transferência de peso para o lado plégico $(70.3 \%)$ e a utilização de feedback visual e auditivo (67.6\%). Conclusáo. No Recife, poucos são os fisioterapeutas neurofuncionais que conhecem a SP. Destes, o nível de informação pode ser considerado insatisfatório e, em alguns aspectos, contraditório.
\end{abstract}

Unitermos. Síndrome de Pusher, Fisioterapia, Hemiparesia.

Citação. Góis CMS, Araújo MCNV, Silva KC, Araújo ATC. Avaliação do Conhecimento dos Fisioterapeutas Neurofuncionais acerca da Síndrome de Pusher.

\begin{abstract}
Objective. To analyze the knowledge level of neurofunctional physiotherapists about Pusher Syndrome (PS). Method. A questionnaire was applied to physiotherapists who execute neurofunctional services in clinics, hospitals and home care services in Recife-PE. Results. Only 37 of the 60 physiotherapists interviewed knew about PS. From this subset of 37 , just $40.5 \%$ had previously done some research about PS. All physiotherapists of this subset pointed to stroke as the principal cause; however, less than 35\% suggested brain tumor and traumatic brain injury. The posterolateral thalamus was indicated by $35.1 \%$ of the physiotherapists as the possible damaged structure. The orthostatic $(62.2 \%)$ and sitting $(21.6 \%)$ postures were identified as the most frequently observed particular behavior of the PS patient. Also, $62.2 \%$ related that the patient resists correction to his posture because of insecurity. The main clinical features reported were displacement of the center of gravity to the paretic side $(59.5 \%)$ and the use of the healthy hemisphere to push (51.4\%). Finally, the therapeutic resources most commonly applied were weight displacement to the paretic side (70.3\%) and audio-visual feedback (67.6\%). Conclusion. In Recife, there are few neurofunctional physiotherapists who know about PS. Among them, the level of information can be considered unsatisfactory and, in some ways, contradictory.
\end{abstract}

Keywords. Pusher Syndrome, Physiotherapy, Hemiparesis.

Citation. Góis CMS, Araújo MCNV, Silva KC, Araújo ATC. Evaluation of the Knowledge about Pusher Syndrome in Neurofunctional Physiotherapists.
Trabalho desenvolvido na Universidade Salgado de Oliveira (UNIVERSO), campus Recife, Recife-PE, Brasil.

1. Fisioterapeuta, Recife-PE, Brasil.

2. Fisioterapeuta, Mestre em Patologia pela Universidade Federal de Pernambuco (UFPE), professora substituta da UFPE, Recife-PE, Brasil.

3. Fisioterapeuta, Mestre em saúde da criança e do adolescente (UFPE), professora titular da Universidade Salgado de Oliveira (UNIVERSO), Campus Recife, Recife-PE, Brasil.
Endereço para correspondência: Karina de Carvalho da Silva Rua das Gardênias, 123 II $^{a}$ etapa Rio Doce CEP 53150-570, Olinda-PE, Brasil.

Tel.: 81 8656-0944

E-mail: karinnacs@yahoo.com.br 


\section{INTRODUÇÃO}

A Síndrome de Pusher (SP), também conhecida como Síndrome do Não-Alinhamento, é considerada uma das mais intrigantes alteraçóes do controle postural vertical que são encontradas em pacientes com lesão encefálica ${ }^{1}$. Embora a causa típica da síndrome esteja associada ao Acidente Vascular Cerebral2,3, o transtorno também pode ser uma consequência de qualquer outra lesão que produza disfunção da rede neural responsável pelo processamento de dados para percepção vertical, como, por exemplo, traumas ou tumores cerebrais ${ }^{2}$. Tais danos neurológicos podem ocorrer com igual frequência tanto no hemisfério direito como no esquerdo ${ }^{4,5}$.

Os pacientes com SP apresentam um comportamento peculiar em relação aos pacientes hemiplégicos típicos ${ }^{1,2,4,6}$ : quando estão sentados ou na posição ortostática ${ }^{1-4,6}$, deslocam seus centros de massa em direção ao hemicorpo acometido, o que torna o corpo lateralmente instável $^{4,7}$. Ademais, tais pacientes resistem à tentativa de correção passiva de sua postura ${ }^{1-4,6}$ no sentido ou por sobre a linha mediana do seu corpo em direção ao lado não afetado, relatando insegurança e medo de cair ${ }^{1,2,4,6}$.

Os achados indicam que a SP está, possivelmente, associada a uma má interpretação da orientação corporal em relação à gravidade ${ }^{4,6,8}$ decorrente de uma alteração na percepção postural vertical ${ }^{3,4,7,9}$. Sendo assim, esses pacientes têm a percepção de que estâo alinhados na vertical quando estão aproximadamente com $20^{\circ}$ de inclinação para o lado plégico ${ }^{4,7,9}$.

Os pacientes com SP apresentam, ainda, um maior comprometimento do nível de consciência corporal e uma maior dificuldade em retornar às atividades de vida diária, quando comparados com pacientes com hemiparesia típica ${ }^{10}$. Dessa forma, acredita-se que o objetivo primordial da fisioterapia para esses pacientes seja oferecer condiçôes para que os mesmos atinjam o mais completamente possível sua funcionalidade ${ }^{4}$, através das estratégias conscientes para controlar a sua postura ${ }^{8}$.

Devido às suas características clínicas peculiares, a SP exige um tratamento diferenciado por parte dos profissionais de reabilitação. Assim sendo, a finalidade do presente estudo foi avaliar o nível de conhecimento dos fisioterapeutas neurofuncionais acerca da SP, bem como determinar os principais achados clínicos e a adequação das intervençôes terapêuticas empregadas pelos mesmos para o tratamento dessa síndrome.

\section{MÉTODO}

\section{Amostra}

A amostra foi constituída por 60 profissionais de fisioterapia, de ambos os sexos, selecionados aleatoriamente a partir da relação emitida pelo Conselho Regional de Fisioterapia e Terapia Ocupacional (CREFITO1), de acordo com os seguintes critérios de inclusão: Fisioterapeutas que realizam atendimento na área neurofuncional em clínicas escolas, clínicas particulares, instituiçóes de ensino superior e serviços de home care na cidade de Recife-PE e foram excluídos da amostra todos os que não trabalham com neurofuncional, ou ainda, aqueles que não possuem experiência no tratamento de paciente com hemiplegia.

\section{Procedimento}

Foi realizado um estudo transversal através da aplicação de um questionário elaborado pelos próprios pesquisadores e dividido em duas partes: a primeira parte do questionário foi composta por perguntas aos dados pessoais do entrevistado (sexo, idade e tempo de formação), além de questão sobre o conhecimento da Síndrome de Pusher. A segunda parte do questionário foi respondida apenas pelos fisioterapeutas que afirmavam conhecer a SP e incluiu questóes específicas sobre a referida síndrome, abrangendo os seguintes tópicos: realização de pesquisas sobre a SP, número de casos atendidos, reconhecimento de possíveis etiologias e sistema lesionado, postura em que mais se observa o comportamento peculiar do paciente com SP, reação do paciente na tentativa de correção passiva de sua postura, características clínicas, posturas utilizadas para tratar o paciente, técnicas e métodos empregados e prognóstico.

A coleta de dados foi realizada no período de julho a setembro de 2009. Os profissionais foram entrevistados nos seus respectivos locais de trabalho e assinaram o Termo de Consentimento Livre e Esclarecido que permitiu a publicação de suas opinióes, garantindo-lhes, entretanto, o sigilo da fonte, conforme determina a resolução $\mathrm{n}^{\circ}$ 196/96 do Conselho Nacional de Saúde (CNS).

O presente estudo foi aprovado pelo Comitê de Éti- 
ca em Pesquisa do Hospital do Câncer (oficio no36/2009), tendo seguido as normas para pesquisa envolvendo seres humanos.

\section{Análise estatística}

Os dados foram inseridos nos programas Microsoft Excel, versão 2000 e SPSS v. 8.0. Após a entrada dos dados, foi realizada análise exploratória para verificação das frequências de todas as variáveis e as medidas de tendência central e de dispersão das variáveis contínuas, através do teste t-Student. Para a análise bivariada foi aplicado o teste Qui-quadrado ou exato de Fisher, quando necessário. Todas as conclusões foram tomadas ao nível de significância de 5\% (p<0,05).

\section{RESULTADOS}

Foram entrevistados 60 profissionais, dos quais a maior parte encontrava-se na faixa etária de até 30 anos $(56.7 \%)$, sendo que a maioria dos fisioterapeutas da amostra tinha entre 1 e 3 anos de conclusão da graduação.

Do total de entrevistados, cerca de 61\% (37/60) informaram conhecer a Síndrome de Pusher. Deste universo, 40.5\% (15/37) informaram ter realizado alguma pesquisa sobre a doença e haviam atendido algum paciente portador da síndrome. As variáveis sexo, idade e tempo de formação não mostraram associação estatisticamente significante em relação ao conhecimento declarado sobre a SP $(\mathrm{p}>0.05)$.

Com relação às perguntas específicas sobre os conhecimentos dos profissionais entrevistados sobre a SP, a respeito da sua etiologia, todos os profissionais que informaram conhecê-la identificaram o acidente vascular cerebral como a principal causa. Entretanto, outras importantes causas da síndrome como o traumatismo cranioencefálico (TCE) e os tumores cerebrais foram referidos por poucos profissionais, (32.4\% e $24.3 \%$, respectivamente).

Em se tratando da estrutura ou sistema lesionado, de todos os fisioterapeutas que afirmaram conhecer a SP, somente 13 (35.1\%) indicaram o local mais frequente da lesão, ou seja, o tálamo póstero-lateral, enquanto $21.6 \%$ (8/37) não souberam responder a esta questão, conforme apresentado no Gráfico 1.

Sobre a postura em que mais se observa o comportamento peculiar do paciente com SP, aproximadamente

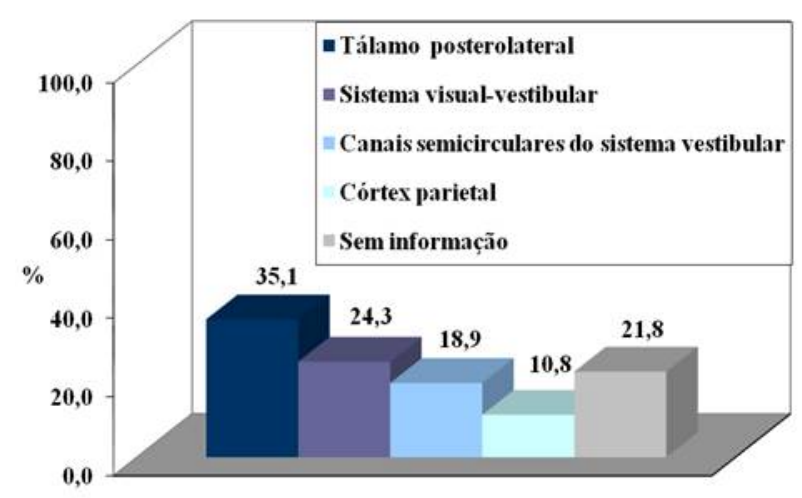

Gráfico 1. Topografia lesional da Síndrome de Pusher, segundo os profissionais que relataram conhecer a doença.

62\% (23/37) dos entrevistados que afirmaram conhecer a doença, informaram ser na postura ortostática que os sintomas mais se manifestam, enquanto 8 profissionais $(21.6 \%)$ relataram que tais sinais são mais frequentes quando o paciente encontra-se sentado (Tabela 1).

De acordo com a Tabela 1 , quanto à reação do paciente na tentativa de correçáo passiva da sua postura pelo fisioterapeuta, $62.2 \%$ (23/37) dos fisioterapeutas informaram que o paciente resiste à correção devido à insegurança. Cerca de 35\% dos profissionais (13/37) não responderam a esta questão.

Quando questionados sobre as características clínicas da SP, os sinais mais citados pelos profissionais foram o deslocamento do centro de gravidade para o lado plégico $(59.5 \%)$ e a utilização do hemicorpo sadio para se empurrar $(51,4 \%)$, conforme apresentado na Tabela 1. Outras características que não fazem parte da síndrome também foram citadas, tais como distúrbios de estereognosia $(8.1 \%)$ e cinestesia $(10.8 \%)$. Cerca de $8 \%$ dos entrevistados que se declararam conhecedores da SP não souberam identificar nenhum sinal da doença (dados não apresentados em tabela).

Quando questionados sobre o tratamento fisioterapêutico dos pacientes com SP, cerca de 59\% (22/37) dos entrevistados informaram que tratariam o paciente na posição vertical e $29.7 \%$ (11/37), na posição horizontal. Com relação às técnicas e métodos terapêuticos utilizados, conforme apresentado no Gráfico 2, as principais condutas relatadas foram: transferência de peso para o lado plégico $(70.3 \%)$ e utilização de feedback visual e auditivo (67.6\%). 
Tabela 1

Características clínicas e comportamento dos pacientes com SP segundo os profissionais que relatam conhecer a doença

\begin{tabular}{ccc}
\hline $\begin{array}{c}\text { Posiçóes em que mais se observa o comportamento } \\
\text { peculiar do paciente com SP }\end{array}$ & n & \% \\
\hline Posiçáo ortostática & 23 & 62.2 \\
Sentado & 8 & 21.6 \\
Deitado & 2 & 5.4 \\
Sem informação & 3 & 8.1 \\
\hline Como paciente reage durante tentativa de & $\mathbf{n}$ & $\%$ \\
correçáo da postura? & 23 & 62.2 \\
\hline Resiste à correção, por insegurança & 3 & 8.1 \\
Indiferente para a correção & 3 & 8.1 \\
Esforça-se para adquirir a posição correta & 13 & 35.0 \\
Sem informaçáo & $\mathbf{n}$ & $\%$ \\
\hline Características clínicas da & 22 & 59.5 \\
Síndrome de Pusher & 19 & 51.4 \\
\hline O centro de gravidade está deslocado para o lado plégico & 11 & 29.7 \\
Utiliza o hemicorpo sadio para se empurrar & 9 & 24.3 \\
Cabeça está inclinada para o lado ipisilateral da lesão & 9 & 24.3 \\
Cervical pode estar rígida & 5 & 13.5 \\
\hline A perna hemiplégica faz rotaçáo medial & &
\end{tabular}

Com relação à influência da SP sobre o prognóstico e evoluçâo do paciente, $54.1 \%$ dos profissionais (20/37) relataram que a mesma é um fenômeno que influencia negativamente o processo de reabilitação.

\section{DISCUSSÃO}

No presente estudo, constatou-se que, apenas 37 fisioterapeutas entrevistados relataram conhecer a Síndrome de Pusher (SP). Destes, menos da metade afirmou ter pesquisado sobre a doença e o mesmo número de fisioterapeutas, afirmou ter atendido pacientes com a síndrome.

Em virtude da importância da SP e de seu impacto sobre o paciente, seria esperado que um percentual maior de profissionais conhecesse essa síndrome, não apenas por possuírem experiência na área específica de neurofuncional, mas principalmente, por tratar-se de uma síndrome amplamente relatada na literatura ${ }^{1}$.

Embora a análise não tenha demonstrado uma relação estatisticamente significante entre o tempo de formação do profissional e o conhecimento da SP, a constatação de que a maioria dos entrevistados apresentava tempo de formação inferior a três anos pode sugerir que as informaçôes acerca da síndrome, oferecidas aos acadê- micos ainda nas universidades, têm se mostrado insuficientes. Este pode ser um fator relevante também para o pequeno relato de ocorrência da doença que, na verdade, poderia ter uma incidência bem maior que a relatada na literatura.

Alguns autores estimam que aproximadamente $10 \%$ dos pacientes que sofrem um acidente vascular cerebral (AVC) apresentam a SP na fase aguda ou subaguda da lesão cerebral ${ }^{10,11}$. Em estudo recente ${ }^{8}$, foi constatado

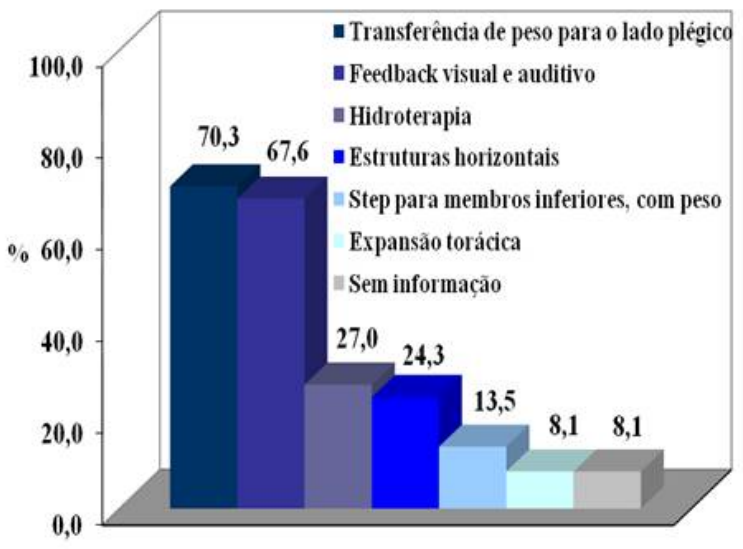

Gráfico 2. Distribuição das condutas terapêuticas utilizadas no tratamento da Síndrome de Pusher. 
que, após um AVC, 20\% dos pacientes apresentaram uma tendência para o desenvolvimento da SP, sendo esse o primeiro estudo acerca deste comportamento na Regiâo Nordeste Brasileira. Em pesquisas anteriores foram relatadas incidências de $28 \%$ da SP em pacientes com a lesão talâmica decorrente de $\mathrm{AVC}^{12}$ e de $63 \%$ em indivíduos com sequelas de acidente vascular em regiōes encefálicas ${ }^{13}$.

Com relação à etiologia da SP, os resultados dessa pesquisa são concordantes com estudos prévios $^{2,6,10,14}$, nos quais o AVC foi citado como a principal causa da SP. Embora outras etiologias tenham sido relatadas, tais como os tumores cerebrais e os traumatismos cranioencefálico (TCE) $)^{2,15,16}$, no presente estudo, poucos fisioterapeutas mencionaram tais eventos e a maioria os descartou como possíveis causas da SP. Essa falta de conhecimento pode gerar consequências importantes para pacientes com TCE e tumor cerebral, que apresentam a SP associada, uma vez que os profissionais podem reforçar os padrôes patológicos da SP e comprometerem a evolução desses pacientes.

Abordando aspectos em relação à estrutura ou sistema lesionado na SP, constatou-se nessa pesquisa que a maioria dos profissionais não sabia informar corretamente a estrutura mais comumente envolvida. Apesar do tálamo póstero-lateral ser a estrutura mencionada com maior frequência na literatura ${ }^{6,7,10,17}$, uma parcela importante dos fisioterapeutas que se declararam conhecedores da SP indicaram, de forma equivocada, o sistema visual-vestibular e os canais semicirculares como os sistemas mais lesionados na SP.

Embora uma das primeiras hipóteses elaboradas para explicar o comportamento peculiar apresentado por pacientes com SP tenha afirmado que tais pacientes apresentariam uma perturbação do sistema visual-vestibular ${ }^{18,19}$, no primeiro estudo em que se investigou essa hipótese, não foram encontrados problemas na recepção dos estímulos por tais sistemas ${ }^{8}$. Pesquisas recentes confirmaram que o sistema visual-vestibular de fato não está relacionado com este fenômeno ${ }^{4,12,15}$.

Por outro lado, existe uma provável relação entre lesões do tálamo posterior com a SP, visto que esta região estaria relacionada com o controle da postura vertical do corpo. Sendo assim, um acometimento nessa área resul- taria em uma inclinação da verticalidade visual subjeti$\mathrm{va}^{12,14}$.

Segundo os resultados desta pesquisa, a principal postura em que os fisioterapeutas observam o comportamento peculiar da SP é a ortostática, corroborando com os achados da literatura ${ }^{4,6,7}$. Porém, verificou-se que apenas um pequeno percentual dos entrevistados indicou também a postura sentada como uma das principais posiçôes em que é possível a identificação dos sinais da $\mathrm{SP}^{2,4,6}$. O conhecimento dessa associação é de suma importância para o fisioterapeuta neurofuncional, uma vez que dá ao mesmo condiçóes de perceber precocemente as alteraçóes no comportamento adotado pelo paciente, proporcionando, assim, um diagnóstico prévio da SP e um tratamento diferenciado para o paciente.

Possivelmente vários pacientes portadores da síndrome não são diagnosticados como tal, pois na fase aguda da lesão náo podem ser submetidos a posturas mais elevadas, dificultando o diagnóstico da síndro$\mathrm{me}^{6,10}$. Assim sendo, fisioterapeutas e enfermeiros podem ser os primeiros a detectar essa desordem diante do leito hospitalar, ao transferirem os pacientes para maca, cadeiras ou durante a realização de tratamento que os coloque em posição sentada ${ }^{6}$. Entretanto, essa identificação precoce dos sinais da SP depende da capacitaçâo de tais profissionais.

O diagnóstico da SP é baseado na Scale Contraversive Pushing ${ }^{12,14,20}$, na qual são avaliados três aspectos distintos do controle postural, um dos quais se refere ao comportamento do paciente, quando da correção passiva da sua postura, enquanto sentado e em pé. No presente estudo, a maioria dos entrevistados relatou que o indivíduo com SP tende a resistir à correção da sua postura em direção à linha mediana, corroborando estudos prévios que descrevem que pacientes portadores da SP demonstram insegurança e medo de cair, quando submetidos à correção passiva de sua postura no sentido ou além da linha mediana do seu corpo em direção ao hemicorpo $\operatorname{sadio}^{1,2,4,7}$.

Tal atitude poderia ser justificada por uma dificuldade de interpretação da orientação corporal em relação à gravidade ${ }^{4,6,8}$ decorrente de uma alteraçáo na percepção postural vertical ${ }^{3,4,7,9}$, de modo que o paciente tem a percepção de que se encontra na posição vertical quando, 
na verdade, apresenta-se com uma inclinação lateral de cerca de $20^{\circ} 8,9,14$. Assim, a resistência apresentada pelo paciente, quando se estimula o posicionamento na linha mediana do seu corpo, impossibilita a postura ortostática, bem como a deambulação e, consequentemente, retarda o tempo de recuperação das suas atividades funcionais ${ }^{8,14}$.

Ao analisar as características clínicas da SP indicadas pelos fisioterapeutas, observou-se que os relatos principais foram o centro de gravidade do paciente deslocado para o lado plégico e a utilização do hemicorpo sadio para empurrar-se, concordando com os achados da literatura que, além dessas, descrevem também como características da SP: coluna cervical rígida com pouca mobilidade; alongamento de toda a musculatura do lado hemiplégico na posição supina; rotação medial da perna hemiplégica e cabeça inclinada para o lado ipisilateral da lesão cerebral ${ }^{1,4}$.

Outras características clínicas que não fazem parte da síndrome também foram citadas pelos entrevistados, tais como distúrbios da estereognosia e cinestesia, hipercinesias, além da utilização do lado plégico para empurrarse. Esse dado reflete, portanto, que os profissionais que se declararam conhecedores da SP, na realidade, foram incapazes de reconhecer acertadamente os sinais indicativos ou não da referida síndrome.

Com relação à melhor postura para o tratamento do paciente com a SP, constatou-se que pouco mais da metade da amostra indicou a postura vertical. Entretanto, vale ressaltar que quase metade dos profissionais, que dizia conhecer a SP, indicou a postura horizontal ou não soube responder a esta questáo. Relatos da literatura também indicam a postura vertical como ideal para o tratamento deste paciente ${ }^{4}$. A finalidade seria fornecer feedback sobre a orientação do corpo em relação à gravidade ${ }^{4,7,21}$, uma vez que tais pacientes apresentam dificuldades em manter o equilíbrio e controle de tronco em situações estáticas e dinâmicas, demonstrando, assim, alteraçóes do alinhamento corpóreo $^{6,20}$.

Quando questionados sobre as condutas terapêuticas utilizadas para o paciente com SP, a maioria dos entrevistados optou pela transferência de peso para o lado plégico e alguns indicaram o uso de estruturas horizontais. Tais indicações não são condutas adequadas ao tratamento, uma vez que a transferência de peso para o lado plégi- co reforçaria o padrão patológico da SP e, consequentemente, o desvio de alinhamento corporal na vertical. Em virtude disso, o ideal é que o fisioterapeuta utilize o treino de transferência de peso para o hemicorpo sadio durante atividades funcionais ${ }^{1,14}$ e que se utilizem estruturas verticais para oferecer um feedback visual sobre a orientação do corpo, compensando, dessa forma, o déficit de má interpretação do corpo em relação à gravidade $e^{4,7,21}$.

Apesar de pacientes com a Síndrome de Pusher não serem capazes de usar espontaneamente um estímulo visual para ajustar sua postura, o controle do corpo na postura ereta pode ser possível através das estratégias conscientes, uma vez que os mesmos preservam a capacidade de alinhar o corpo ao eixo vertical com a ajuda de pistas visuais ${ }^{8}$.

A utilização dessas condutas foi confirmada em uma pesquisa ${ }^{22}$ que descreveu um método de tratamento baseado na transferência de peso para a perna não parética na transição da postura sentada para de pé; step com peso para membros inferiores; treino de marcha; subir e descer escadas; feedback visual e auditivo. Os autores relataram redução dos sintomas da Síndrome de Pusher e observaram, ainda, que o uso do feedback visual e auditivo proporcionou efeitos positivos imediatos na obtençáo da posição bípede independente.

A SP é considerada um fenômeno transitório ${ }^{13,23}$ normalmente associado a um bom prognóstico e que parece não influenciar negativamente os resultados da reabilitaçấo ${ }^{7}$. Segundo alguns autores, os sinais diminuem algumas semanas após o evento neurológico e desaparecem em cerca de seis semanas a três meses ${ }^{13,14,21}$. No presente estudo, entretanto, a maioria dos profissionais relatou que a SP influencia negativamente a reabilitação do paciente, proporcionando um prognóstico ruim. Esse dado merece especial atenção, pois uma grande parte desses pacientes muitas vezes é interpretada como não tendo boa indicação para a reabilitação e, desta forma, o tratamento específico acaba sendo retardado, devido ao conhecimento insuficiente dos profissionais de saúde ${ }^{1}$.

Assim, as condutas contraditórias referidas pelos fisioterapeutas entrevistados, bem como sua inadequada interpretação quanto ao prognóstico dos pacientes com SP, colocam em dúvida o nível de conhecimento desses profissionais, o que pode prejudicar a evolução do pa- 
ciente, uma vez que o mesmo necessita de um tratamento específico e diferente daquele aplicável a pacientes com hemiplegia típica.

\section{CONCLUSÃO}

Diante dos resultados apresentados, observou-se que, embora a maioria dos fisioterapeutas entrevistados tenha afirmado conhecer a Síndrome de Pusher, esses mesmos profissionais entraram em contradição quanto a diversos aspectos da doença, inclusive com relação à conduta terapêutica. Dessa forma, ficou evidente que o conhecimento sobre esse importante fenômeno é insuficiente, o que pode prejudicar o tratamento dos pacientes, uma vez que o entendimento dos mecanismos etiológicos e clínico-patológicos é essencial para a elaboração de estratégias adequadas de reabilitação.

Por outro lado, esse escasso conhecimento dos fisioterapeutas, que lidam diretamente com o controle postural, pode torná-los incapazes de identificar a SP em seus pacientes e este fator pode estar associado com uma prevalência declarada do fenômeno aquém da realidade vivenciada na clínica por profissionais experientes.

Assim torna-se evidente a necessidade de divulgação dessa síndrome por parte das instituições de ensino superior e cursos de fisioterapia neurofuncional, de modo a proporcionar uma base para que os fisioterapeutas possam diagnosticar mais facilmente a SP, bem como elaborar estratégias mais eficazes de tratamento.

\section{REFERÊNCIAS}

1.Davies PM. Síndrome de Não Alinhamento (Síndrome de Pusher). In: MIR Assessoria Editorial Ltda. Passos a Seguir (Um manual para o tratamento da hemiplegia do adulto). Dr. Nelson Gomes de Oliveira. São Paulo: Editora Manole, 1996, p.279-98.

2.Santos-Pontelli TEG, Pontes-Neto OM, Colafêmina JF, Araujo DB, Santos AC, Leite JP. Contraversive Pushing in non-stroke patients. J Neurol 2004;251:1324-8.

http://dx.doi.org/10.1007/s00415-004-0532-y

3. Cardoen S, Santens P. Posterior pusher syndrome: A report of two cases. Clin Neurol Neurosurg 2010;112:347-9.

http://dx.doi.org/10.1016/j.clineuro.2009.12.007

4.Ticini LF, Klose U, N囚gele T, Karnath HO. Perfusion Imaging in Pusher Syndrome to Investigate the Neural Substrates Involved in Controlling Upri- ght Body Position. PLoS ONE. 2009;4:e5737.

http://dx.doi.org/10.1371/journal.pone.0005737

5.Karnath HO, Ferber S, Dichgans J. The neural representation of postural control in humans. Proc Natl Acad Sci. 2000;97:13931-6.

http://dx.doi.org/10.1073/pnas.240279997

6.Karnath HO. Pusher Syndrome: a frequent but little-know disturbance of body orientation perception. J Neurol 2007;341:514-24.

7.Premoselli S, Cesana L, Cerri C. Pusher syndrome in stroke: clinical, neuropsychological and neurophysiological investigation. Eur Med Phys 2001;37:143 -51. 8.Pedersen PM, Wandel A, Jorgensen HS, Nakayama H, Raaschou HO, Olsen TS. Ipsilateral pushing in stroke: incidence, relation to neuropsychological symptoms, and impact on rehabilitation the Copenhagen stroke study. Arch Phys Med Rehabil. 1996; 77:25-8.

http://dx.doi.org/10.1016/S0003-9993(96)90215-4

9.Karnath HO, Johannsen L, Broetz D, Küker W . Posterior thalamic hemorrhage induces "pusher syndrome”. Neurology 2005;64:1014-9.

http://dx.doi.org/10.1212/01.WNL.0000154527.72841.4A

10.Danells CJ, Black SE, Gladstone DJ, McIlroy WE. Poststroke "Pushing": natural history and relationship to motor and functional Recovery. Stroke 2004;35:2873-8.

http://dx.doi.org/10.1161/01.STR.0000147724.83468.18

11.Melo MEFA. Prevalência e perfil anátomo-clínico-funcional da Síndrome do Não-Alinhamento em pacientes vítimas de Acidente Vascular Encefálico (Dissertação). Recife: UFPE, 2009,72p.

12.Karnath HO, Broetz D. Understanding and treating "pusher syndrome". Phys Ther. 2003;83:1119-25.

13.Karnath HO, Ferber S, Dichgans J. The origin of controversies pushing. Neurology. 2000;55:1298-304.

14.Karnath HO, Johannsen L, Broetz D, Ferber S, Dichgans J. Prognosis of contraversive pushing. J Neurol. 2002;249:1250-3.

http://dx.doi.org/10.1007/s00415-002-0824-z

15.Santos-Pontelli TEG, Pontes-Neto OMP, Colafêmina J, Araújo DB, Santos AC, Leite JP. Controle postural na síndrome de pusher: influência dos Canais semicirculares laterais. Rev Bras Otorrinolaringol. 2005;71:448-52.

16.Santos-Pontelli TEG, Pontes-Neto OM, Colafêmina JF, Araújo DB, Santos AC, Leite JP. Pushing behavior and hemiparesis: which is critical for functional recovery in pusher patients? case report. Arq Neuropsiquiatr 2007;65:536-9. http://dx.doi.org/10.1590/S0004-282X2007000300035

17.Johannsen L, Broetz D, Naegele T, Karnath HO. "Pusher syndrome" following cortical lesions that spare the thalamus. J Neurol 2006;253:455-63.

http://dx.doi.org/10.1007/s00415-005-0025-7

18.Vibert D, Haüsler R, Safran AB. Subjective visual vertical in peripheral unilateral vestibular diseases. J Vestib 1999;145:152-9.

19.Brandt T, Dieterich M, Danek A. Vestibular cortex lesions affect the perception of verticality. Ann Neurol 1994; 403:412-35.

20.Saj A, Honoré J, Coello Y, Rousseaux M. The Visual Vertical in the Pusher Syndrome: influence of Hemispace and Body Position. J Neurol 2005;252:885-91.

http://dx.doi.org/10.1007/s00415-005-0716-0

21.Broetz D, Johannsen L, Karnath HO. Time course of "pusher syndrome" under visual feedback treatment. Physiother Res Int 2004;9:138-43.

http://dx.doi.org/10.1002/pri.314

22.Paci M, Nannetti L. Physiotherapy for pusher behaviour in a patient with post-stroke hemiplegic. J Rehabil Med 2004;36:183-5.

http://dx.doi.org/10.1080/16501970410029762

23. Cecatto RB, Almeida CI. Síndrome de Pusher após Acidente Vascular Encefálico: relato de caso. Acta Fisiatr 2008;15:195-201. 